NASA Technical Memorandum 107632

p. 26

\title{
LINEAR SYSTEM IDENTIFICATION VIA BACKWARD-TIME OBSERVER MODELS
}

Jer-Nan Juang and Minh Phan

June 1992

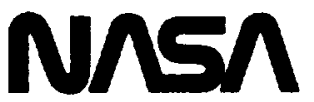

National Aeronautics and

Space Administration

Langley Research Center

Hampton, Virginia 23665

(NASA-TY-107632) LINEAP SYSTEM
I TENTIFICATICN VIA PACKWARO-TIME OBSERVER
MPUELS (NASA) 26 P

$N 92-30214$ 
-

-

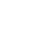




\title{
Linear System Identification via Backward-time Observer Models
}

\author{
Jer-Nan Juang ${ }^{1}$ and Minh Phan ${ }^{2}$ \\ NASA Langley Research Center, Hampton, VA
}

\begin{abstract}
This paper presents an algorithm to compute the Markov parameters of a backwardtime observer for a backward-time model from experimental input and output data. The backward-time observer Markov parameters are decomposed to obtain the backward-time system Markov parameters (backward-time pulse response samples) for the backward-time system identification. The identified backward-time system Markov parameters are used in the Eigensystem Realization Algorithm to identify a backward-time state-space model, which can be easily converted to the usual forward-time representation. If one reverses time in the model to be identified, what were damped true system modes become modes with negative damping, growing as the reversed time increases. On the other hand, the noise modes in the identification still maintain the property that they are stable. The shift from positive damping to negative damping of the true system modes allows one to distinguish these modes from noise modes. Experimental results are given to illustrate when and to what extent this concept works.
\end{abstract}

\section{Introduction}

Identification is the process of developing or improving a mathematical model of a physical system using experimental data to describe the input, output and noise relationship. A tremendous number of system identification techniques are available. Techniques to identify a model from input and output data typically contain two steps. First, a family of candidate models is chosen and then the particular member in this family is determined which satisfactorily describes the observed data based on some error criterion such as minimizing the measurement residuals due to the input and output noises. One of the most popular models is ARX model ${ }^{1}$ where AR refers to the autoregressive part (related to output data) and X

\footnotetext{
${ }^{1}$ Principal Scientist, Spacecraft Dynamics Branch, Fellow AIAA.

'Senior Research Engineer, Lockheed Engineering \& Sciences Co., Hampton, Va., Member AIAA.
} 
refers to the exogeneous part (related to input data). In particular, the forward-time ARX model in the time domain is nothing but a linear finite difference equation describing that the current output measurement can be estimated from past output and input data. On the other hand, the backward-time ARX model means that the current output measurement can be estimated by the future output and input data. The ARX coefficients in the linear finite difference equation can be easily computed from input and output data.

Insights into physical mechanisms of the system can usually be incorporated more easily into state-space models than the ARX models just described above. In the state-space form the relationship between the input, output, and noise data is written as a system of firstorder differential equations using an auxiliary state vector. Note that it is difficult to directly compute a state-space model from input and output data. The state-space models can be constructed directly from the ARX coefficients using standard canonical forms including companions form, controllable/observable canonical form, Jordan canonical form, etc. One common problem is that an estimate of system order is required to be known a priori. Since the model order is generally unknown in practice, a trial and error process is required to find the best model order. In general, it results in a considerably overspecified model order particularly when the data contains considerable noise. Overspecification of the model order increases accuracy but discrimination of the system and computational portions of the models can be difficult. Recently, novel techniques referred to as backward methods using the backward-time model have been introduced in Refs. 2 and 3 to aid in the mode discrimination process. If one reverses time in the model to be identified, the damped true system modes become unstable, growing as the reversed time increases. On the other hand, the noise modes in the identification still maintain the property that they are stable. This is intuitively reasonable. If the data set is sufficiently long, an unstable noise mode would contribute to the pulse response data that grow unbounded as the time step in the data set increases. This is inconsistent with the expected contribution of noise in data. The shift from positive damping to negative damping of the true system modes allows one to distinguish these modes from noise modes.

Aside from the problems of model order using standard canonical forms, numerical sensitivity is known to result from using the state space model whose elements are taken directly from the ARX model coefficients. The interested reader is directed to Refs. 4-6 for discussions of numerical sensitivities of companion forms, controllable/observable canonical forms, etc. The backward method introduced in this paper avoids numerically sensitive canonical forms by constructing a state-space realization from backward-time Markov parameters. This 
method uses the observer-based approaches which are developed in Refs. 7-10 and referred to as the Observer/Kalman filter identification (OKID) method. The primary step is to use an observer to produce the backward-time Markov parameters (backward-time pulse response samples) from input and output data. The backward-time Markov parameters are then used in the Eigensystem Realization Algorithm (ERA) ${ }^{11-12}$ to construct a backward-time statespace model for its modal parameter estimation. As a by-product, a backward-time observer is obtained to be used for noise characterization. The identified backward-time state space model can be easily converted to the usual (forward-time) state-space representation.

This paper starts by writing the relationship between the input and output histories in terms of the deterministic backward-time ARX model. Formulations are derived to compute the system backward-time Markov parameters and the backward-time observer gain from the backward-time ARX coefficients. The second part of this paper is to extend the deterministic case to the stochastic case with process and measurement noises. This will result in a backward-time ARMAX model where MA refers to the moving average part which is related to the residual of the measurement equation. The ARMAX model describes not only the deterministic but also the stochastic components of the response. The relationship betwen the backward-time observer and the Kalman filter is discussed. Experimental results in comparison with the forward OKID method ${ }^{7-10}$ are given to illustrate the validity of the backward method presented in this paper. The experimental results are obtained from a 10-bay truss structure having two accelerometers and two thrusters.

\section{Deterministic Backward-time ARX Model}

Consider a discrete multivariable linear system described by the state equation

$$
x(k+1)=A x(k)+B u(k)
$$

and the measurement equation

$$
y(k)=C x(k)+D u(k)
$$

where $x(k) \in R^{n}, y(k) \in R^{m}, u(k) \in R^{r}$, and $k$ is the time index. Premultiplying Eq. (1) by $A^{-1}$ and solving for $x(k)$ yield

$$
x(k)=A^{-1} x(k+1)-A^{-1} B u(k)
$$


This is a backward-time model in the sense that the current state $x(k)$ is determined by the future state $x(k+1)$ and the current input $u(k)$. It is not a natual model and cannot be used in real time implementation. However, it can be very useful for modal parameter identification of a system model, which will be shown in the following. Substracting and adding the term $G y(k+1)$ in Eq. (3) produces

$$
\begin{aligned}
& x(k)=A^{-1} x(k+1)-A^{-1} B u(k)+G y(k+1)-G y(k+1) \\
& =A^{-1} x(k+1)-A^{-1} B u(k)+G C x(k+1)+G D u(k+1)-G y(k+1) \\
& =\left(A^{-1}+G C\right) x(k+1)+\left[\begin{array}{lll}
-A^{-1} B & G D & -G
\end{array}\right]\left[\begin{array}{c}
u(k) \\
u(k+1) \\
y(k+1)
\end{array}\right]
\end{aligned}
$$

This is a backward-time observer model which observes the current state $x(k)$ using the future state $x(k+1)$ and the observer gain $G$ multiplied by a combination of inputs and outputs. The advantage of using the observer model instead of the original backward-time model is that the observer gain can be chosen so that the observer state matrix $\left(A^{-1}+G C\right)$ is as stable as desired. Define

$$
\begin{aligned}
& \bar{A}=A^{-1}+G C, \tilde{B}=-A^{-1} B \\
& \bar{B}=\left[\begin{array}{lll}
\tilde{B} & G D & -G
\end{array}\right], \bar{v}(k)=\left[\begin{array}{c}
u(k) \\
u(k+1) \\
y(k+1)
\end{array}\right]
\end{aligned}
$$

Using the above definition, Eq. (4) can be shortened as

$$
x(k)=\bar{A} x(k+1)+\bar{B} \bar{v}(k)
$$

Equation (6) looks identical to a typical linear discrete model except that it is backward in time. From Eqs. (2) and (6), the mapping between the input and output data can be established by the following equation

$$
y(k)=C \bar{A}^{p} x(k+p)+C \bar{A}^{p-1} \bar{B} \bar{v}(k+p-1)+\ldots+C \bar{B} \bar{v}(k)+D u(k)
$$

This equation computes an estimate of the current measurement $y(k)$ using the $p$-step-future state $x(k+p)$, current and future inputs, $u(k), \ldots, u(k+p)$, and future output measurements, $y(k+1), y(k+2), \ldots, y(k+p)$. Let $G$ and $p$ be chosen such that

$$
\bar{A}^{i}=\left(A^{-1}+G C\right)^{i}=0 ; i \geq p
$$

Physically, it means that the gain matrix $G$ makes the backward-time observer state matrix $A^{-1}+G C$ becomes deadbeat after $p$ steps. It implies that the current output measurement 
can be fully estimated by the future $p$ inputs and outputs (see Eq. (7)) without any future state involved. The smaller the $p$ is the shorter the number of the future inputs and outputs is needed to estimate the current measurement. However, there is a lower limit for the integer $p$ which is related to the order of the system and the number of outputs. For the single-output observable case, there always exists a gain $G$ which makes the state matrix $A^{-1}+G C$ becomes deadbeat as long as the chosen integer $p$ is greater than or equal to the order of the system matrix $A$. For the multiple-output observable case, if $p$ is chosen such that the product of $p$ and the number of outputs is greater than or equal to the system order, a gain $G$ always exists to make the observer state matrix $A^{-1}+G C$ becomes deadbeat after $p$ steps. The reader is directed to Ref. 10 for the proof of the above statements.

Substitution of $\bar{B}$ and $v$ defined in Eq. (5) into Eq. (7) yields

$$
\begin{aligned}
& y(k)+C G y(k+1)+\ldots+C \bar{A}^{p-1} G y(k+p) \\
& =(D+C \tilde{B}) u(k)+(C \bar{A} \tilde{B}+C G D) u(k+1)+\ldots \\
& +\left(C \bar{A}^{p-1} \tilde{B}+C \bar{A}^{p-2} G D\right) u(k+p-1)+C \bar{A}^{p-1} G D u(k+p)
\end{aligned}
$$

or

$$
\begin{aligned}
& y(k)+\alpha_{1} y(k+1)+\ldots+\alpha_{p} y(k+p) \\
& =\beta_{0} u(k)+\beta_{1} u(k+1)+\ldots+\beta_{p} u(k+p)
\end{aligned}
$$

where

$$
\begin{aligned}
& \alpha_{i}=C \bar{A}^{i-1} G ; i=1,2, \ldots, p \\
& \beta_{i}=C \bar{A}^{i} \tilde{B}+C \bar{A}^{i-1} G D ; i=1,2, \ldots, p-1 \\
& \beta_{0}=D+C \tilde{B} ; \beta_{p}=C \bar{A}^{p-1} G D
\end{aligned}
$$

Equation (10) is known to be a backward-time ARX model. At this moment, the relationship between the backward-time state space model, Eq. (3), and the backward-time ARX model becomes clear. Given the system matrices $A, B, C, D$, and the observer gain matrix $G$, the backward-time ARX mdel, Eq. (10), can be easily derived by using Eq. (11). On the other hand, if the coefficients $\alpha_{i}$ and $\beta_{i}(i=1,2, \ldots, p)$ of the ARX model are directly computed from the input and output data, are the system matrices $A, B, C, D$ realizable from these coefficients? This question will be answered in the following. 
Let Eq. (10) be rewritten in the following matrix form

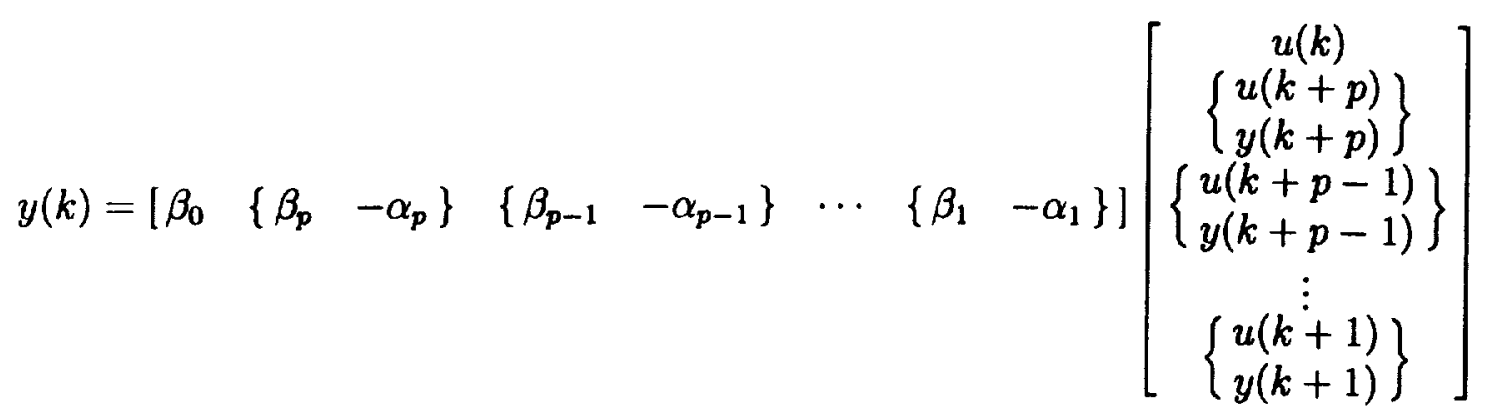

Now define

$$
\begin{aligned}
& y=\left[\begin{array}{lllll}
y(0) & y(1) & y(2) & \cdots & y(\ell-p)
\end{array}\right]
\end{aligned}
$$

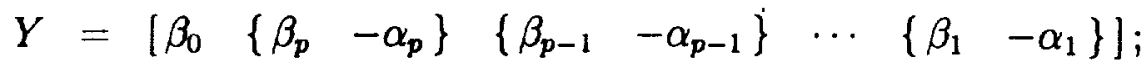

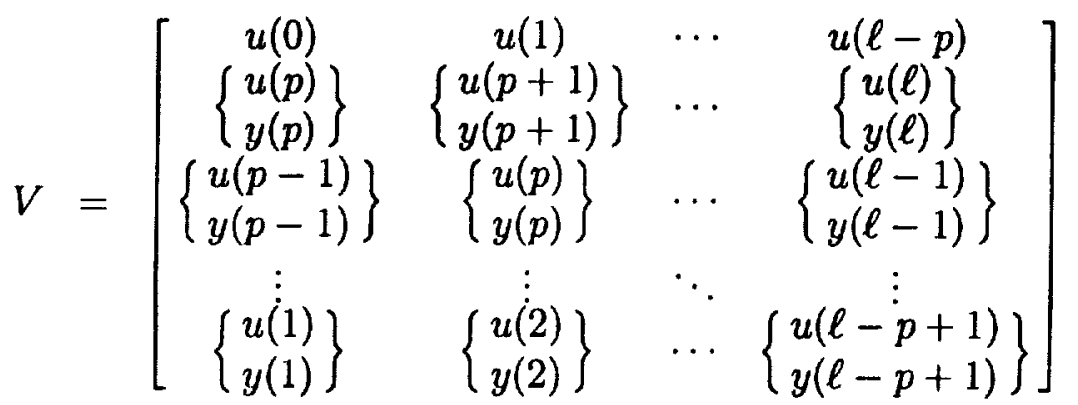

The matrix $Y$ of dimension $m \times[r+(m+r) p]$ contains the unknown ARX coefficients $\alpha_{i}$ and $\beta_{i}(i=1,2, \ldots, p)$, whereas the matrices $y$ of dimension $m \times(\ell-p+1)$ and $V$ of dimension $[r+(m+r) p] \times(\ell-p+1)$ contain the input and output data. Making use of Eq. (12) and the definition of Eq. (13) produces

$$
y=Y V
$$

which yields

$$
Y=y V^{\dagger}
$$

where $\dagger$ means the pseudo-inverse of $V$. Equation (15) solves for the unknown ARX coefficients $\alpha_{i}$ and $\beta_{i}(i=1,2, \ldots, p)$. Having computed these ARX coefficients, the system matrices $A, B, C, D$ can be constructed as follows.

Using Eq. (11) yields

$$
\begin{aligned}
& D=-\alpha_{p}^{-1} \beta_{p} \\
& C \tilde{B}=\beta_{0}-D \\
& C \bar{A}^{i} \tilde{B}=\beta_{i}-\alpha_{i} D
\end{aligned}
$$


From Eqs. (11) and (16), one obtains the following Markov-like parameter sequences

$$
Y_{o}=\left[\begin{array}{llll}
C G & C \bar{A} G & \cdots & C \bar{A}^{p-1} G
\end{array}\right]
$$

and

$$
Y_{s}=\left[\begin{array}{lllll}
D & C \tilde{B} & C \bar{A} \tilde{B} & \cdots & C \bar{A}^{p-1} \tilde{B}
\end{array}\right]
$$

All system information is embedded in the sequences $Y_{o}$ and $Y_{s}$. The next step is to extract the system Markov parameters from the two sequences for the identification of the system matrices $A, B, C, D$. By simply applying the same approach as in Ref. 10, the sequences defined in Eqs. (17) and (18) can be decoupled to produce the following combined Markov parameters,

$$
Y_{c}=\left[\begin{array}{lll}
D & C\left[\begin{array}{ll}
\tilde{B} & G
\end{array}\right] \quad C A^{-1}\left[\begin{array}{ll}
\tilde{B} & G
\end{array}\right] \cdots \quad \cdots A^{-k}\left[\begin{array}{ll}
\tilde{B} & G
\end{array}\right]
\end{array}\right]
$$

where $k$ is an arbitrary integer which can be as large as desired. The sequence $Y_{c}$ is defined as the combined Markov parameters to describe the input-output map of a backward-time observer model as defined in Eqs. (4) in combination with the measurement equation, Eq. (3). The combined Markov parameter sequence can be used in conjunction with the ERA algorithm in Ref. 11 or 12 to realize a set of matrices

$$
\left[A^{-1} \tilde{B} C D G\right]
$$

Using the relationship $\tilde{B}=-A^{-1} B$, the forward-time representation can be computed

$$
[A B C D G]
$$

with $B=-A \tilde{B}$. The operator "inverse", $A^{-1}$, shifts all the eigenvalues of $A$ from inside the unit circle to outside the unit circle. In other words, the stable system modes will become unstable modes by the inverse operator.

\section{Stochastic Backward-time ARMAX Model}

Equation (11) is a deterministic backward-time ARX model representing the state space model given in Eqs. (1) and (2). In the following development, an extension to the case with process and measurement noises will be made. This will result in a backward-time ARMAX model that describes not only the deterministic but also the stochastic components of the response. Consider the system with process and measurement noises given below,

$$
\begin{array}{ll}
x(k+1) & =A x(k)+B u(k)+w_{1}(k) \\
y(k) & =C x(k)+D u(k)+w_{2}(k)
\end{array}
$$


where $x(i) \in R^{n}, y(i) \in R^{m}, u(i) \in R^{r}$. Ideally, the process noise $w_{1}(k)$ and measurement noise $w_{2}(k)$ are two uncorrelated zero-mean stationary white noise processes. From Kalman filter theory, there exists a Kalman filter gain $K$ such that the system given in Eq. (20) is equivalent to

$$
\begin{array}{ll}
\hat{x}(k+1) & =A \hat{x}(k)+B u(k)+K \varepsilon(k) \\
y(k) & =C \hat{x}(k)+D u(k)+\varepsilon(k)
\end{array}
$$

where $\varepsilon(k)$ is a residual sequence which is white and uncorrelated with the measurements, and whose Euclidean norm is minimized. To derive a backward-time model for the above system, premultiply Eq. (21) by $A^{-1}$ and solve for $\hat{x}(k)$,

$$
\hat{x}(k)=A^{-1} \hat{x}(k+1)-A^{-1} B u(k)-A^{-1} K \varepsilon(k)
$$

This is a backward-time stochastic state space model in the sense that the current state $\hat{x}(k)$ is described by the future state $\hat{x}(k+1)$, the current input $u(k)$, and the current residual $\varepsilon(k)$. If the original system is stable then the backward-time system will be unstable. To derive the backward-time ARMAX model, add and subtract the term $G y(k+1)$ in Eq. (22),

$$
\begin{aligned}
\hat{x}(k)= & A^{-1} \hat{x}(k+1)-A^{-1} B u(k)-A^{-1} K \varepsilon(k)+G y(k+1)-G y(k+1) \\
= & A^{-1} \hat{x}(k+1)-A^{-1} B u(k)-A^{-1} K \varepsilon(k)+G C \hat{x}(k+1)+G D u(k+1) \\
& +G \varepsilon(k+1)-G y(k+1)
\end{aligned}
$$

With the definitions as given in Eq. (5), Eq. (23) can be simplified as

$$
\begin{aligned}
\hat{x}(k) & =\bar{A} \hat{x}(k+1)+\tilde{B} u(k)+\tilde{K} \varepsilon(k)+G D u(k+1)+G \varepsilon(k+1)-G y(k+1) \\
& =\bar{A} \hat{x}(k+1)+\left[\begin{array}{lll}
\tilde{B} & G D & -G
\end{array}\right]\left[\begin{array}{c}
u(k) \\
u(k+1) \\
y(k+1)
\end{array}\right]+\left[\begin{array}{ll}
\tilde{K} & G
\end{array}\right]\left[\begin{array}{c}
\varepsilon(k) \\
\varepsilon(k+1)
\end{array}\right]
\end{aligned}
$$

where $\tilde{K}=-A^{-1} K$. The expression for the output $y(k)$ becomes

$$
\begin{aligned}
y(k)= & C \bar{A} \hat{x}(k+1)+(C \tilde{B}+D) u(k)+C G D u(k+1)-C G y(k+1) \\
& +(I+C \tilde{K}) \varepsilon(k)+C G \varepsilon(k+1)
\end{aligned}
$$

The estimated state vector $\hat{x}(k+1)$ can be eliminated by successive substitution of Eq. (24) into Eq. (25). For example,

$$
\begin{aligned}
\hat{x}(k+1) & =\bar{A} \hat{x}(k+2)+\tilde{B} u(k+1)+\tilde{K} \varepsilon(k+1) \\
& +G D u(k+1)+G \varepsilon(k+2)-G y(k+2)
\end{aligned}
$$


Equation (25) then becomes

$$
\begin{aligned}
y(k) & =C \bar{A}^{2} \hat{x}(k+2)+(C \tilde{B}+D) u(k)+(C \bar{A} \tilde{B}+C G D) u(k+1)+C \bar{A} G D u(k+2) \\
& -C G y(k+1)-C G y(k+1)-C \bar{A} G y(k+2) \\
& +(I+C \tilde{K}) \varepsilon(k)+(C \bar{A} \bar{K}+C G) \varepsilon(k+1)+C \bar{A} G \varepsilon(k+2)
\end{aligned}
$$

Repeating the substitution $p-1$ times, and making use of the imposed deadbeat condition for $G$, i.e.,

$$
\bar{A}^{i}=\left(A^{-1}+G C\right)^{i}=0 \quad i \geq p
$$

yields the following backward-time description relating the current and future outputs to current and future inputs and residuals,

$$
\begin{aligned}
y(k)+ & C G y(k+1)+C \bar{A} G y(k+2)+\cdots+C \bar{A}^{p-1} G y(k+p) \\
= & (D+C \tilde{B}) u(k)+(C \bar{A} \bar{B}+C G D) u(k+1)+\cdots \\
& +\left(C \bar{A}^{p-1} \tilde{B}+C \bar{A}^{p-2} G D\right) u(k+p-1)+C \bar{A}^{p-1} G D u(k+p)+e(k)
\end{aligned}
$$

where $e(k)$ is a colored residual which is related to the white residual of the Kalman filter $\varepsilon(k)$ by the relation

$$
\begin{aligned}
e(k)= & (I+C \tilde{K}) \varepsilon(k)+(C \bar{A} \tilde{K}+C G) \varepsilon(k+1)+\cdots \\
& +\left(C \bar{A}^{p-1} \tilde{K}+C \bar{A}^{p-2} G\right) \varepsilon(k+p-1)+C \bar{A}^{p-1} G \varepsilon(k+p)
\end{aligned}
$$

Define $\alpha_{i}, \beta_{i}$ as in Eqs. (11)

$$
\begin{aligned}
\alpha_{i} & =C \bar{A}^{i-1} G ; i=1,2, \cdots, p \\
\beta_{i} & =C \bar{A}^{i-1} \tilde{B}+C \bar{A}^{i-1} G D ; i=1,2, \cdots, p-1 \\
\beta_{0} & =D+C \tilde{B} ; \beta_{p}=C \bar{A}^{p-1} G D
\end{aligned}
$$

and $\gamma_{i}$ as

$$
\begin{aligned}
& \gamma_{i}=C \bar{A}^{i} \tilde{K}+C \bar{A}^{i-1} G ; i=1,2, \cdots, p-1 \\
& \gamma_{0}=I+C \tilde{K} ; \gamma_{p}=C \bar{A}^{p-1} G
\end{aligned}
$$

Equations (29) and (30) become

$$
\begin{aligned}
y(k) & +\alpha_{1} y(k+1)+\cdots+\alpha_{p} y(k+p) \\
& =\beta_{0} u(k)+\beta_{1} u(k+1)+\cdots+\beta_{p} u(k+p)+e(k)
\end{aligned}
$$

and

$$
e(k)=\gamma_{0} \varepsilon(k)+\gamma_{1} \varepsilon(k+1)+\cdots+\gamma_{p} \varepsilon(k+p)
$$


Equation (33) above is now referred to as a backward-time ARMAX model. The coefficients of the backward-time ARMAX model are expressed in terms of the state space matrices $A, B, C, D$, a backward-time observer gain matrix $G$, and the (forward-time) Kalman filter gain $K$. Observe that the deterministic portion of the model described by the coefficients $\alpha_{i}, \beta_{i}$ is the same as the backward-time ARX model derived in Eq. (10).

\section{System Identification using a Backward-time ARMAX Model}

The problem of system identification using this model structure consists of three basic steps:

1. Determination of the model coefficients $\alpha_{i}, \beta_{i}$, and $\gamma_{i}$ from input and output data.

2. Computation of the appropriate Markov parameters from the model coefficients $\alpha_{i}, \beta_{i}$, and $\gamma_{i}$.

3. Realization of the system state space model and the corresponding observer gains from the Markov parameters.

Each of the three steps will be described below. First, it is convenient to rewrite Eq. (33) in the following form,

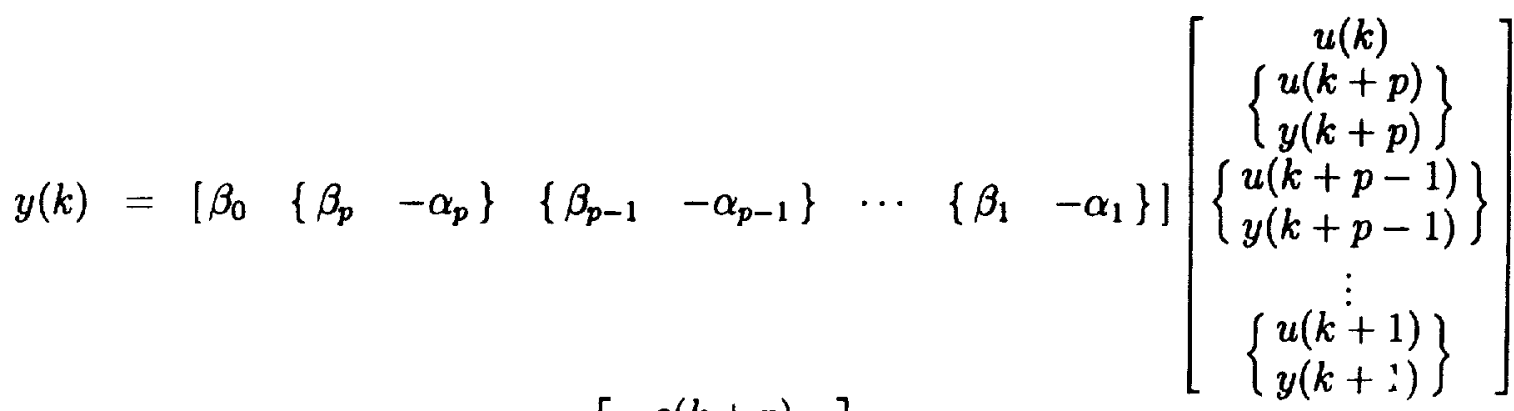

$$
\begin{aligned}
& +\left[\begin{array}{llll}
\gamma_{p} & \gamma_{p-1} & \cdots & \gamma_{1}
\end{array}\right]\left[\begin{array}{c}
\varepsilon(k+p) \\
\varepsilon(k+p-1) \\
\vdots \\
\epsilon(k+1)
\end{array}\right]+\gamma_{0} \varepsilon(k)
\end{aligned}
$$

For a given input-output time history, Eq. (35) can be written as

$$
\begin{aligned}
& y=Y V+e \\
& e=\Lambda W+\zeta \\
& \zeta=\gamma_{0} \varepsilon
\end{aligned}
$$


where $y, Y$, and $V$ are given in Eq. (13), and $e, \zeta \varepsilon \Lambda, W$ are defined as

$$
\begin{aligned}
e & =\left[\begin{array}{lllll}
e(0) & e(1) & e(2) & \cdots & e(\ell-p)
\end{array}\right] \\
\zeta & =\left[\begin{array}{lllll}
\zeta(0) & \zeta(1) & \zeta(2) & \cdots & \zeta(\ell-p)
\end{array}\right] \\
\varepsilon & =\left[\begin{array}{lllll}
\varepsilon(0) & \varepsilon(1) & \varepsilon(2) & \cdots & \varepsilon(\ell-p)
\end{array}\right] \\
\Lambda & =\left[\begin{array}{lllll}
\gamma_{p} & \gamma_{p-1} & \cdots & \gamma_{1}
\end{array}\right] \\
W & =\left[\begin{array}{cccc}
\varepsilon(p) & \varepsilon(p+1) & \cdots & \varepsilon(\ell) \\
\varepsilon(p-1) & \varepsilon(p) & \cdots & \varepsilon(\ell-1) \\
\vdots & \vdots & \ddots & \vdots \\
\varepsilon(1) & \varepsilon(2) & \cdots & \varepsilon(\ell-p+1)
\end{array}\right]
\end{aligned}
$$

At this point, the following observation can be made. The ordinary least-squares solution, which was derived from a purely deterministic consideration as given in Eq. (15), minimizes the norm of the colored residual $\varepsilon$, whereas the true solution minimizes the white residual $\varepsilon$. The least-squares solution, Eq. (15), is the solution of a linear problem, viz., $y=Y V$. This is in contrast with the parameter estimation problem considered here, namely,

$$
y=Y V+\Lambda W+\gamma_{0} \varepsilon
$$

where in addition to the parameters in $Y$, neither $\Lambda, \gamma_{0}$ nor the residual in $W$ are known. This is a non-linear problem since it involves products of the unknowns in $\Lambda W$ and $\gamma_{0} \varepsilon$. When no closed-form solution is available, typical solution to this type of problem is iterative in nature. This approach has been considered previously in the forward-time observer/Kalman filter identification problem, which produces an improved solution under certain conditions when the ordinary least-squares solution is not optimal. The readers are referred to Ref. 13 for further details. Examining the structure of Eq. (36) suggests the following iterating procedure, which starts with the ordinary least-squares solution $Y_{L S}$ obtained from the backward-time ARX approach.

Step 1: Compute the least-squares solution $Y_{L S}$ and the resultant least-squares error,

$$
\begin{aligned}
& Y_{L S}=y V^{T}\left(V V^{T}\right)^{-1} \\
& e_{L S}=y-Y_{L S} V
\end{aligned}
$$

Step 2: Use the initial least-squares error $e_{L S}$ as an initial estimate of the white residual $\varepsilon$ denoted by $\varepsilon_{(1)}$, i.e., let $\varepsilon_{(1)}=e_{L S}$, and compute an estimate of backward-time ARMAX model coefficients in $Y$ and $\Lambda$, denoted by $Y_{(1)}, \Lambda_{(1)}$,

$$
\left[Y_{(1)}, \Lambda_{(1)}\right]=y_{(1)} U_{(1)}^{T}\left(U_{(1)} U_{(1)}^{T}\right)^{-1}
$$


where

$$
\begin{aligned}
y_{(1)} & =\left[\begin{array}{lllll}
y(0) & y(1) & y(2) & \cdots & y(\ell-2 p)
\end{array}\right] \\
U_{(1)} & =\left[\begin{array}{c}
V \\
W_{(1)}
\end{array}\right] \\
W_{(1)} & =\left[\begin{array}{cccc}
\varepsilon_{(1)}(p) & \varepsilon_{(1)}(p+1) & \cdots & \varepsilon_{(1)}(\ell-p) \\
\varepsilon_{(1)}(p-1) & \varepsilon_{(1)}(p) & \cdots & \varepsilon_{(1)}(\ell-p-1) \\
\vdots & \vdots & \ddots & \vdots \\
\varepsilon_{(1)}(1) & \varepsilon_{(1)}(2) & \cdots & \varepsilon_{(1)}(\ell-2 p+1)
\end{array}\right]
\end{aligned}
$$

The resultant least-squares error, denoted by $\zeta_{(1)}$, is computed from

$$
\zeta_{(1)}=y_{(1)}-Y_{(1)} V-\Lambda_{(1)} W_{(1)}
$$

Note that the parameters in $Y_{(1)}$ and $\Lambda_{(1)}$ do not include the first coefficient of the MA portion of the model denoted by $\gamma_{0}$. This coefficient is estimated in step 3 .

Step 3: Compute an estimate of the first coefficient of the MA portion of the backward-time ARMAX model, denoted by

$$
\gamma_{0,(1)}=\zeta_{(1)} \varepsilon_{(1)}^{T}\left[\varepsilon_{(1)} \varepsilon_{(1)}^{T}\right]^{-1}
$$

At this step, a complete set of the backward-time ARMAX model coefficient estimates is obtained in $\gamma_{0,(1)}, Y_{(1)}$, and $\Lambda_{(1)}$.

Step 4: Compute a new estimate of the whitened residual, denoted by $\varepsilon_{(2)}$, from

$$
\varepsilon_{(2)}=\gamma_{0,(1)}^{-1} \zeta_{(1)}
$$

Step 5: Return to step 2 with the new whitened residual $\varepsilon_{(2)}$ obtained in step 5 to compute new estimates of $Y$ and $\Lambda$, denoted by $Y_{(2)}, \Lambda_{(2)}$, and then to step 3 and step 4 to compute new estimates of $\gamma_{0}$, denoted by $\gamma_{0,(2)}$, and a new whitened residual denoted by $\varepsilon_{(3)}$.

The above iterating cycle is repeated and the results examined after each cycle to determine a reasonable stopping point. Observe that after each cycle is completed, the data record to be used in the next cycle is shortened by $p$ points. This is due to the backward-time nature of the ARMAX model which requires knowledge of $p$ future data points to compute the current response. This is in contrast with the forward-time model where the initial condition can be neglected after the transient has decayed. In the backward-time model, 
however, one cannot neglect future data points unless they are known to have decayed to negligible values. Specifically, given a data record of $\ell$ points, a backward-time model of order $p$ can only estimate the first $\ell-p$ data points. Provided the data record to be used in the above iterating procedure is sufficiently long, the above shortening of the data record does not present a problem.

One can compute the Markov parameter sequences of the backward-time observer and system

$$
\begin{aligned}
Y_{o} & =\left[\begin{array}{lllll}
C G & C \bar{A} G & \ldots & C \bar{A}^{p-1} G
\end{array}\right] \\
Y_{s} & =\left[\begin{array}{lllll}
D & C \tilde{B} & C \bar{A} \tilde{B} & \cdots & C \bar{A}^{p-1} \tilde{B}
\end{array}\right]
\end{aligned}
$$

by Eqs. (11) and (16). Furthermore, since

$$
\begin{aligned}
& C \tilde{K}=\gamma_{0}-I \\
& C \bar{A}^{i} \tilde{K}=\gamma_{i}-\alpha_{i}
\end{aligned}
$$

the following Markov parameter sequence can also be computed

$$
Y_{f}=\left[\begin{array}{llll}
C \tilde{K} & C \bar{A} \tilde{K} & \cdots & C \bar{A}^{p-1} \tilde{K}
\end{array}\right]
$$

The backward-time system and observer information is contained in the sequences $Y_{o}$ and $Y_{s}$, whereas the noise information is contained in the sequence $Y_{f}$. The rest of the identification procedure parallels the previous development with the exception that the identified noise model is being carried in the sequence $Y_{f}$. Using the sequences defined in Eqs. (42) and (44), the following combined Markov parameter sequence can be computed,

$$
Y_{c}=\left[\begin{array}{llllllll}
D & C & {\left[\begin{array}{lll}
\tilde{B} & G & \tilde{K}
\end{array}\right]} & C A^{-1} \tilde{B} & \cdots & C A^{-k}\left[\begin{array}{lll}
\tilde{B} & G & \tilde{K}
\end{array}\right]
\end{array}\right]
$$

where $k$ is an arbitrary integer which can be as large as desired. The extra Markov parameters are computed by invoking the prescribed deadbeat condition

$$
C \bar{A}^{i}\left[\begin{array}{lll}
\tilde{B} & G & \tilde{K}
\end{array}\right]=0 \quad i \geq p
$$

Application of the Eigensystem Realization Algorithm (ERA) to the sequence defined in Eq. (45) will produce a realization of the backward-time state space model denoted by

$$
\left[\begin{array}{llllll}
A^{-1} & \tilde{B} & C & D & G & \tilde{K}
\end{array}\right]
$$

Using the relationship, $\tilde{B}=-A^{-1} B, \tilde{K}=-A^{-1} K$, the forward-time system and observer/Kalman filter model represented by

$$
\left[\begin{array}{llllll}
A & B & C & D & G & K
\end{array}\right]
$$


can be easily computed. Note that $G$ is a backward-time observer gain for the system matrix inverse $A^{-1}, K$ is the Kalman filter gain, and the matrices $A, B, C, D$ form the state space model of the system being identified.

\section{Experimental Results}

The developed method is applied to data obtained from the truss structure shown in Fig. 1 at NASA Langley Research Center. The L-shaped aluminum truss is oriented such that its long section is in a vertical direction extending 90 inches. The short section, 20 inches long, is horizontal and is clamped at the free end to a steel rigid plate. The square cross section is 10 inches by 10 inches. Two cold air jet thrusters, located at the beam tip, serve as actuators for excitation and control. Each thruster has a maximum thrust of 2.2 lbs. Two servo accelerometers located at a corner of the square cross section provides the in-plane tip acceleration measurements. In addition, an offset weight of 30 pounds is added to enhance the dynamic coupling between the two principal axes, and to lower the structure fundamental frequency. For identification, the truss is excited using random inputs to both thrusters. The acceleration signals are filtered by a three-pole analog Bessel filter with a break frequency of $20 \mathrm{~Hz}$. The input-output signals are sampled at $250 \mathrm{~Hz}$ and recorded for system identification. The following examples illustrate the application of system identification method developed in this paper using both backward-time ARX and backward-time ARMAX model approaches. All results are obtained using the System/Observer/Controller Identification Toolbox ${ }^{14}$ developed at NASA Langley Research Center.

Identification Using Backuard-time ARX Model

An input-output data record of 2000 points is used in the computation. The order of the ARX model is set to be 10, i.e., $p=10$. Equation (15) is used to compute the backwardtime observer Markov parameters from which the Markov parameters of the backward-time system are computed. Application of ERA produces a backward-time state space model which is then converted to a forward-time representation. Recall that in the backward-time model, stable system modes appear unstable, whereas the noise modes remain stable. When converted to a forward-time representation, the system modes become stable and the noise modes will become unstable and can be eliminated. In other words, this process is simply model reduction by modal truncation where the unstable noise modes (in the forward-time representation) are discarded. The final reduced order model so obtained contains only the stable system modes that are identified by the method. 
Figures $2 \mathrm{a}$ and $2 \mathrm{~b}$ show the reconstructed responses for both outputs using a 6 -order state space model obtained by this approach. In these and the following figures, the solid curves are the actual responses that are used in the identification procedure, and the dashed curves represent the reconstruction. The quality of the reconstruction shows how well the identified model represents the actual system in terms of its ability to reproduce the actual responses when driven by the same input time histories. For convenience, the backward-time identification method presented in this paper is referred to as backward-time OKID, whereas the method formulated in Ref. 10 is referred to as forward-time OKID. When appropriate, the type of model used (ARX or ARMAX model) is also indicated. As a baseline comparison, the reconstruction plots using the 18-th order model obtained by the forward OKID method with the same set of data are also shown in Figs. 3a and $3 \mathrm{~b}$.

\section{Identification Using A Backward-time ARMAX Model}

Recall that the primary purpose of using the ARMAX model structure is to improve results obtained from the ARX model alone by modelling the noise dynamics. In order to show the relative merit of the iterative procedure using the backward-time ARMAX approach, the data record that was used to obtain the results presented in Figs. 2 and 3 is cut short to 1000 points, resulting in a worsened model obtained by the ARX approach. This is reflected in its poor ability to reproduce the data as shown in Figures $4 \mathrm{a}$ and $4 \mathrm{~b}$.

The iterative procedure is then applied to model the noise dynamics and correct the original model. Figures $5 \mathrm{a}$ and $5 \mathrm{~b}$ show the reconstruction plots after one cycle of iteration. The results deteriorate but they are explainable since the procedure starts with the assumption that the least-squares residual obtained with the original ARX solution is the same as the whitened residual of the ARMAX model. Figures $6 \mathrm{a}$ and $6 \mathrm{~b}$ show the reconstruction plots after two cycles of iteration. The identification results are clearly improved. Further improvement can be obtained with additional iterating cycles. However, experiences have shown that later cycles produce little improvement in the results.

As another comparison of the relative performance between the different methods, Table 1 shows the identified frequencies and damping factors for each of the respective cases considered above. In theory, if one uses a backward-time approach then the unstable modes in the forward-time representation can be attributed to noise and eliminated from the final models. Comparison with forward-time model results, however, suggests that these modes could in fact be actual modes, but they are not identified by the backward method due to factors such as slight non-linearities, non-ideal noise conditions in the test data. Inclusion of these modes in the model would bring the final state space model order from 6 to 18 , which 
is the model used to generate Figs. 2a and $2 \mathrm{~b}$. Table 1 also shows the orders of the state space models that are used to compute the reconstruction plots shown previously, and the norm of the residual using the identified model for each respective method. On the other hand, a careful examination of the data shows that there is little improvement in the quality of the reconstruction by including these modes in the final state space models. Taking into account that the data record is short, this is a case where it is not clear to distinguish a system mode which is weakly present in the data from a noise mode. The backward-time approach, however, gives a strong indication about the true system modes. This is evident from the consistencies in the modes identified from both backward-time and forward-time approaches.

\section{Concluding Remarks}

A system identification procedure using a backward-time approach has been formulated. A fundamental contribution of this paper is the connection of the state space model to backward-time finite difference models, which include both a backward-time deterministic auto-regressive model and a backward-time stochastic autoregressive moving average model. In the former case, the connection is established in terms of a backward-time observer, and in the latter case it is established in terms of a backward-time observer and a Kalman filter. The deterministic formulation is considerably simpler in that it is a linear parameter estimation problem. The stochastic approach, which requires modelling of the noise dynamics, can provide improved results over those obtained from the deterministic approach. This is accomplished at the expense of an iterating solution to a non-linear parameter estimation problem. A key feature of the backward-time models is that stable system modes appear unstable whereas the noise modes remain stable. This offers an opportunity to distinguish system modes from noise modes. Since the final state space models contain only the stronger stable modes, the backward method generally yields models of smaller order when compared with models obtained from a forward method. It is advantageous to use the backward method in conjunction with a forward method, so that the effective modes in the final model can be better evaluated. Since the model structure of the backward-time model is very different from that of the forward-time model, examination of the results provided by the two approaches can produce valuable insights into the system being identified. 


\section{References}

${ }^{1}$ Ljung, L., System Identification: Theory for the User, Prentice-Hall, Inc., Englewood Cliffs, New Jersey, 1987.

${ }^{2}$ Hollkamp, J.J. and Batill, S.M., "Automated Parameter Identification and Order Reduction for Discrete Series Models," AIAA Journal, Vol. 29, No. 1, 1991.

${ }^{3}$ Hollkamp, J.J. and Batill, S.M., "Structural Identification Using Order Overspecified TimeSeries Models," to appear in Journal of Dynamics Systems, Measurement, and Control, 1992.

${ }^{4}$ Wilkinson, J.H., Rounding Errors in Algebraic Processes, Prentice-Hall, Inc., Englewood Cliffs, New Jersey, 1963.

${ }^{5}$ Wilkinson, J.H., The Algebraic Eigenvalue Problem, Oxford University Press, 1965.

${ }^{6}$ Grace, A., Laub, A.J., Little, J.N., and Thompson, C., Control System Toolbox: User's Guide, The MathWorks, Inc., October 1990.

${ }^{7}$ Chen, C.-W., Huang, J.-K., Phan, M., and Juang, J.-N., "Integrated System Identification and Modal State Estimation for Control of Large Flexible Space Structures," Journal of Guidance, Control, and Dynamics, Vol. 15, No. 1, pp. 88-95, Jan.-Feb. 1992.

${ }^{8}$ Phan, M., Juang, J.-N., and Longman, R.W., "Identification of Linear Multivariable Systems from a Single Set of Data by Identification of Observers with Assigned Real Eigenvalues," Proceedings of the AIAA 32nd Structures, Structural Dynamics and Materials Conference, Baltimore, MD., April, 1991; also to appear in Journal of the Astronautical Sciences.

${ }^{9}$ Phan, M., Horta, L.G., Juang, J.-N., and Longman, R.W., "Linear System Identification Via an Asymptotically Stable Observer," Proceedings of the AIAA Guidance, Navigation and Control Conference, New Orleans, Louisiana, Aug. 1991; also to appear in Journal of Optimization Theory and Application. An expanded study is to appear as NASA Technical Paper 3164, 1992.

${ }^{10}$ Juang, J.-N., Phan, M., Horta, L.G., and Longman, R.W.., "Identification of Observer/Kalman Filter Markov Parameters: Theory and Experiments," Proceedings of the AIAA Guidance, Navigation and Control Conference, New Orleans, Louisiana, Aug. 1991, and also to appear in Journal of Guidance, Control and Dynamics.

${ }^{11}$ Juang, J.-N., and Pappa, R.S., "An Eigensystem Realization Algorithm for Modal Parameter Identification and Model Reduction," Journal of Guidance, Control, and Dynamics, Vol. 8, No. 5, Sept.-Oct. 1985, pp. 620-627. 
${ }^{12}$ Juang, J.-N., Cooper, J.E., and Wright, J.R., "An Eigensystem Realization Algorithm Using Data Correlations (ERA/DC) for Modal Parameter Identification," Control-Theory and Advanced Technology, Vol. 4, No. 1, 1988, pp. 5-14.

${ }^{13}$ Phan, M., Horta, L.G., Juang, J.-N., and Longman, R.W., "Improvement of Observer/Kalman Filter Identification (OKID) by Residual Whitening," AIAA Guidance, Navigation and Control Conference, Hilton Head, South Carolina, Aug. 1992.

${ }^{14}$ Juang, J.-N., Horta, L.G., and Phan, M.,, "User's Guide for System/Observer/ Controller Identification Toolbox," NASA Technical Memorandum 107566, 1992.

Table 1: Identified frequencies and damping values of the truss structure.

\begin{tabular}{|c|c|c|c|c|c|}
\hline $\mathrm{N}=$ & Mode & $\begin{array}{l}\text { OKID_f }{ }^{t} \\
\text { ARX } \\
2000 \text { pts }\end{array}$ & $\begin{array}{l}\text { OKID_b* } \\
\text { ARX } \\
2000 \mathrm{pts}\end{array}$ & $\begin{array}{l}\text { OKID_b* } \\
\text { ARX } \\
1000 \mathrm{pts}\end{array}$ & $\begin{array}{l}\text { OKID_b* } \\
\text { ARMAX } \\
1000 \text { pts }\end{array}$ \\
\hline \multirow{9}{*}{$\begin{array}{l}\text { Freq. } \\
(\mathrm{Hz} .)\end{array}$} & 1 & 5.87 & 5.89 & 5.92 & 5.89 \\
\hline & 2 & 7.29 & 7.30 & 7.33 & 7.33 \\
\hline & 3 & 19.91 & 18.82 & 18.54 & 15.86 \\
\hline & 4 & 46.60 & 46.64 & 46.26 & 46.14 \\
\hline & 5 & 48.61 & 48.57 & 48.61 & 48.64 \\
\hline & 6 & 64.41 & 64.54 & 64.15 & 60.98 \\
\hline & 7 & 73.76 & 73.76 & 73.43 & 74.37 \\
\hline & 8 & 105.63 & 105.51 & 104.01 & 99.83 \\
\hline & 9 & 110.90 & 110.97 & 111.43 & 113.52 \\
\hline \multirow{9}{*}{$\begin{array}{l}\text { Damp. } \\
(\%)\end{array}$} & 1 & 0.65 & 0.61 & 0.64 & 0.50 \\
\hline & 2 & 0.47 & 0.29 & -0.17 & 0.89 \\
\hline & 3 & 61.52 & 0.91 & 0.76 & 21.73 \\
\hline & 4 & 2.08 & -2.32 & -2.93 & -0.85 \\
\hline & 5 & 1.53 & -0.77 & -0.95 & -0.63 \\
\hline & 6 & 9.33 & -0.944 & -7.80 & -0.56 \\
\hline & 7 & 0.74 & -1.61 & -2.19 & -0.55 \\
\hline & 8 & 10.24 & -10.21 & -9.29 & -11.27 \\
\hline & 9 & 5.12 & -5.26 & -5.40 & -2.11 \\
\hline $\begin{array}{l}\text { State Space } \\
\text { Model Order }\end{array}$ & & 18 & 6 & 4 & 6 \\
\hline Residual & & 0.256 & 0.317 & 0.673 & 0.318 \\
\hline
\end{tabular}

† Forward-time OKID, * Backward-time OKID 


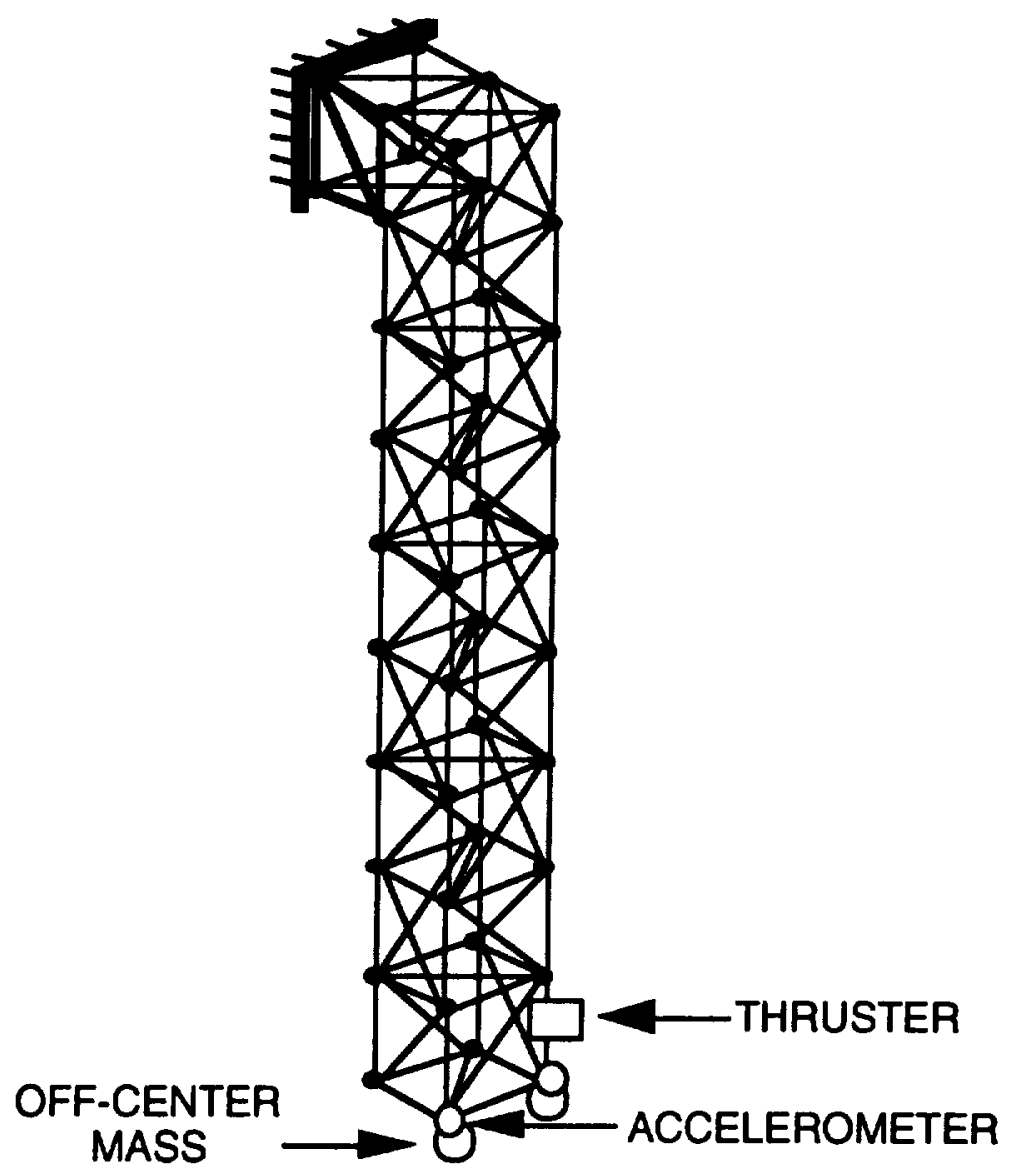

Fig. 1: Truss Structure Test Configuration. 


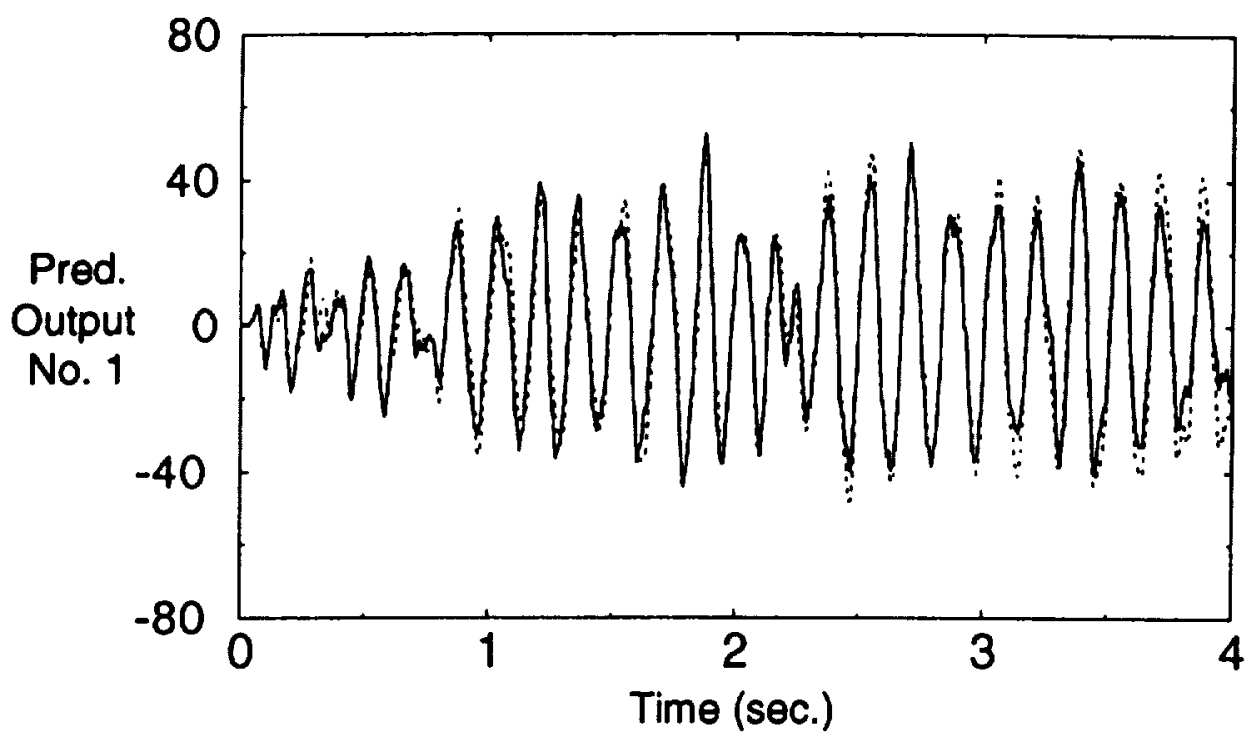

Fig. 2a: Actual vs. reconstructed response for the first output (- Test, - - Reconstruction): Backward-time OKID via an ARX model of order $p=10$ with 2000 data points.

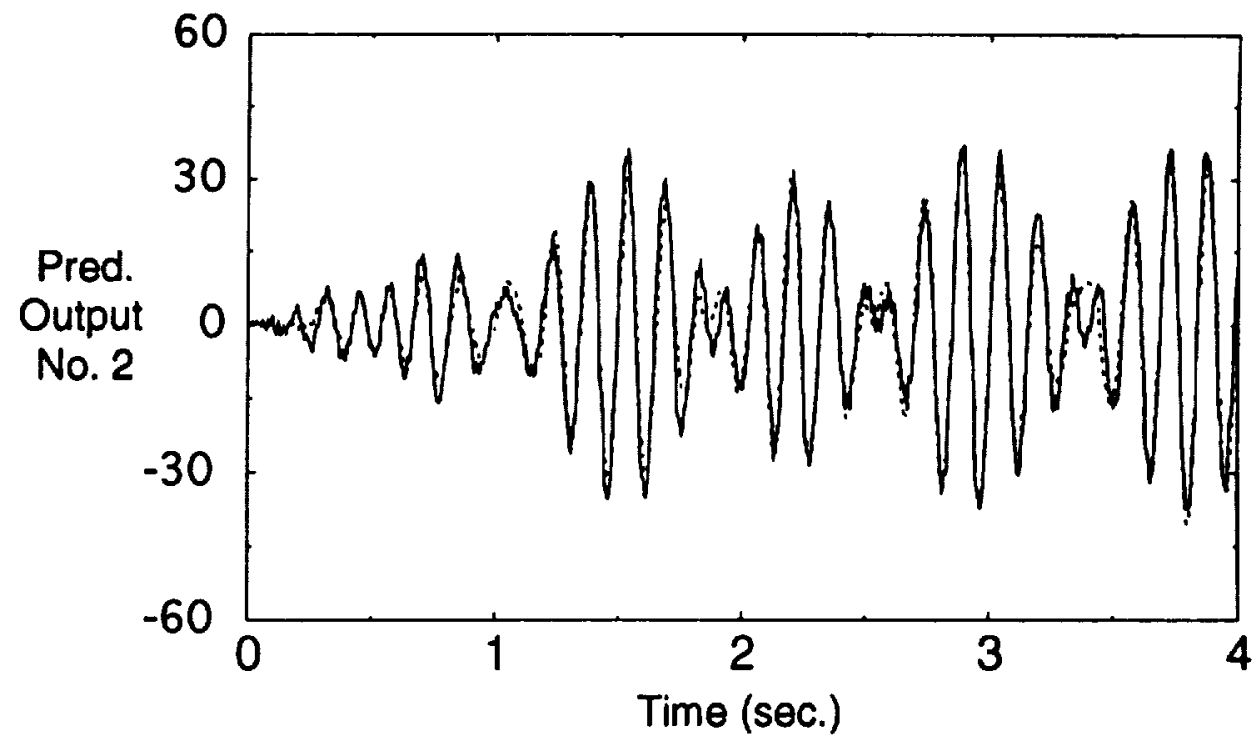

Fig. 2b: Actual vs. reconstructed response for the second output (- Test, - - Reconstruction): Backwardtime OKID via an ARX model of order $p=10$ with 2000 data points. 


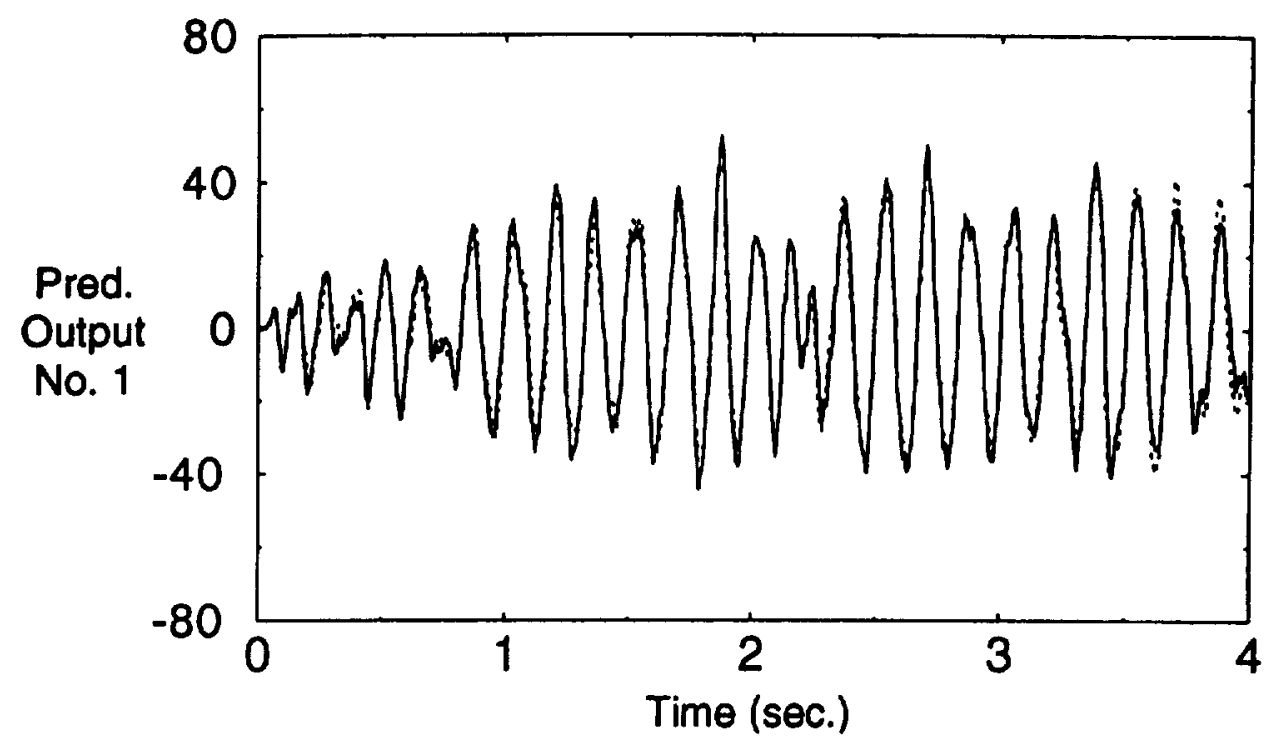

Fig. 3a: Actual vs. reconstructed response for the first output (- Test, - - Reconstruction): Forward OKID via an ARX model of order $\mathrm{p}=10$ with 2000 data points.

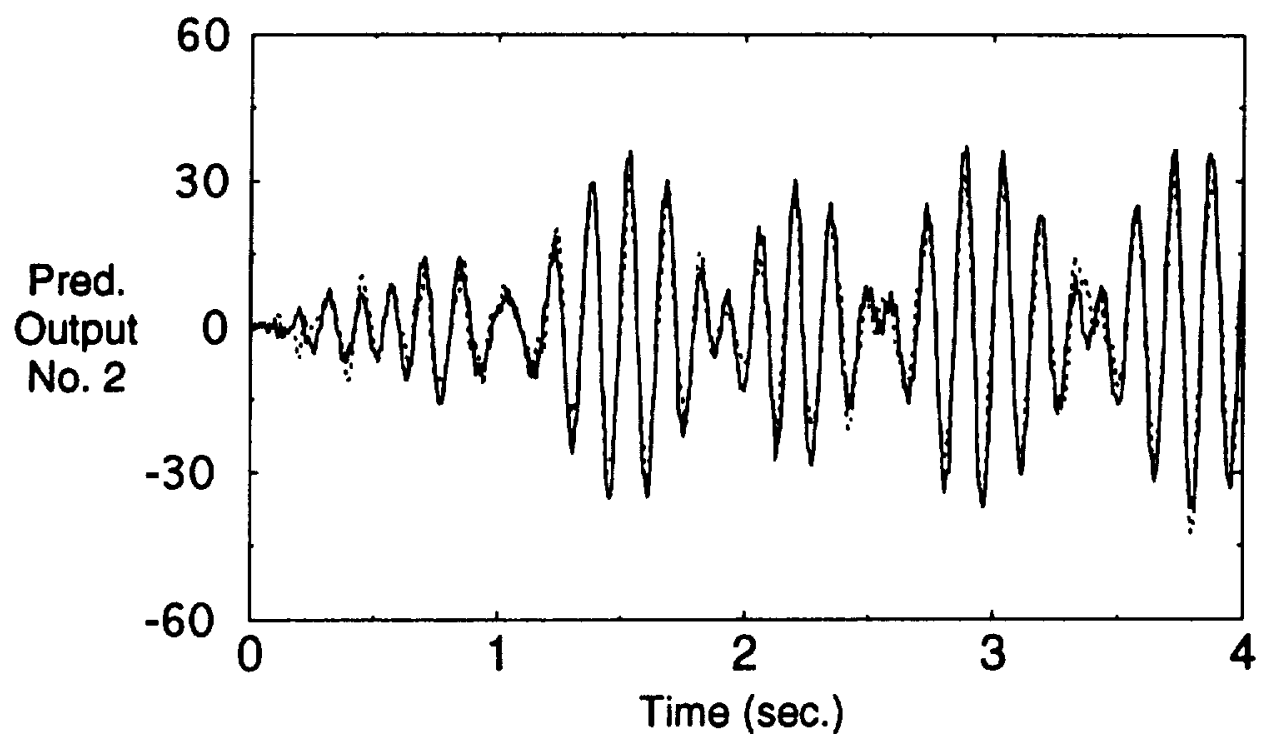

Fig. 3b: Actual vs. reconstructed response for the second output ( - Test, - - Reconstruction): Forward OKID via an ARX model of order $p=10$ with 2000 data points. 


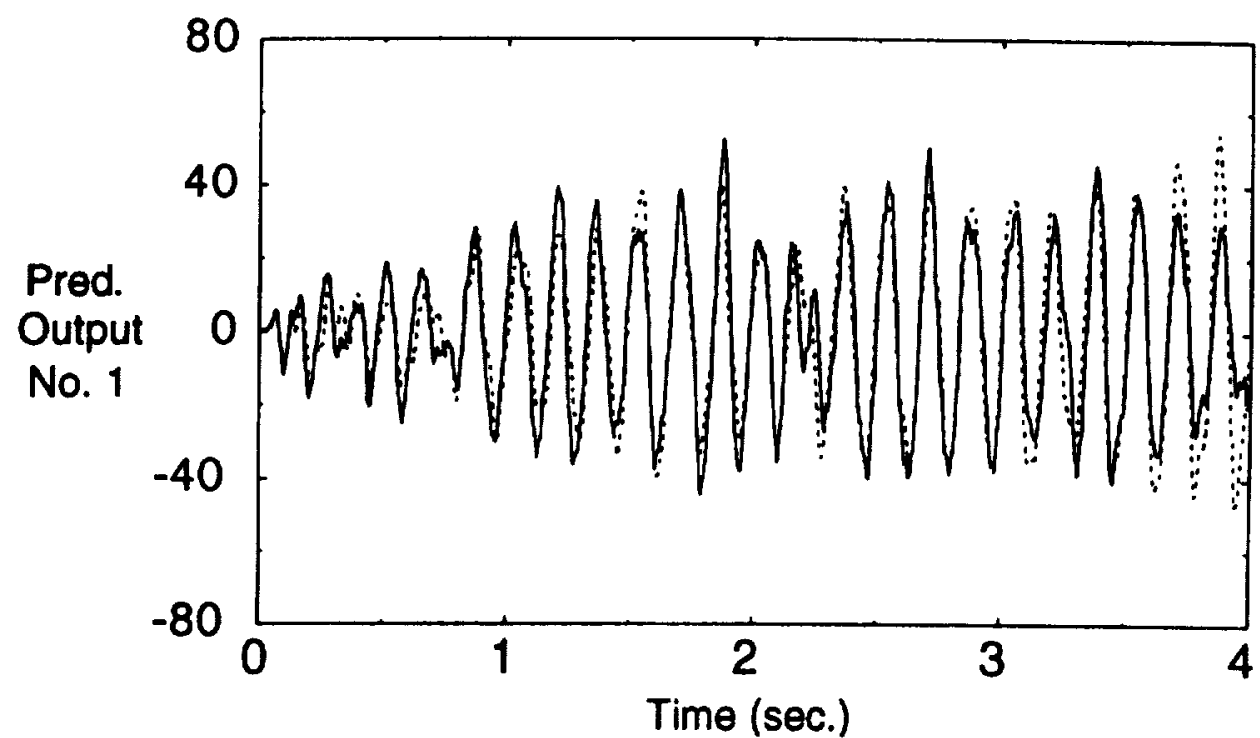

Fig. 4a: Actual vs. reconstructed response for the first output (- Test, - - Reconstruction): Backward OKID via an ARX model of order $p=10$ with 1000 data points.

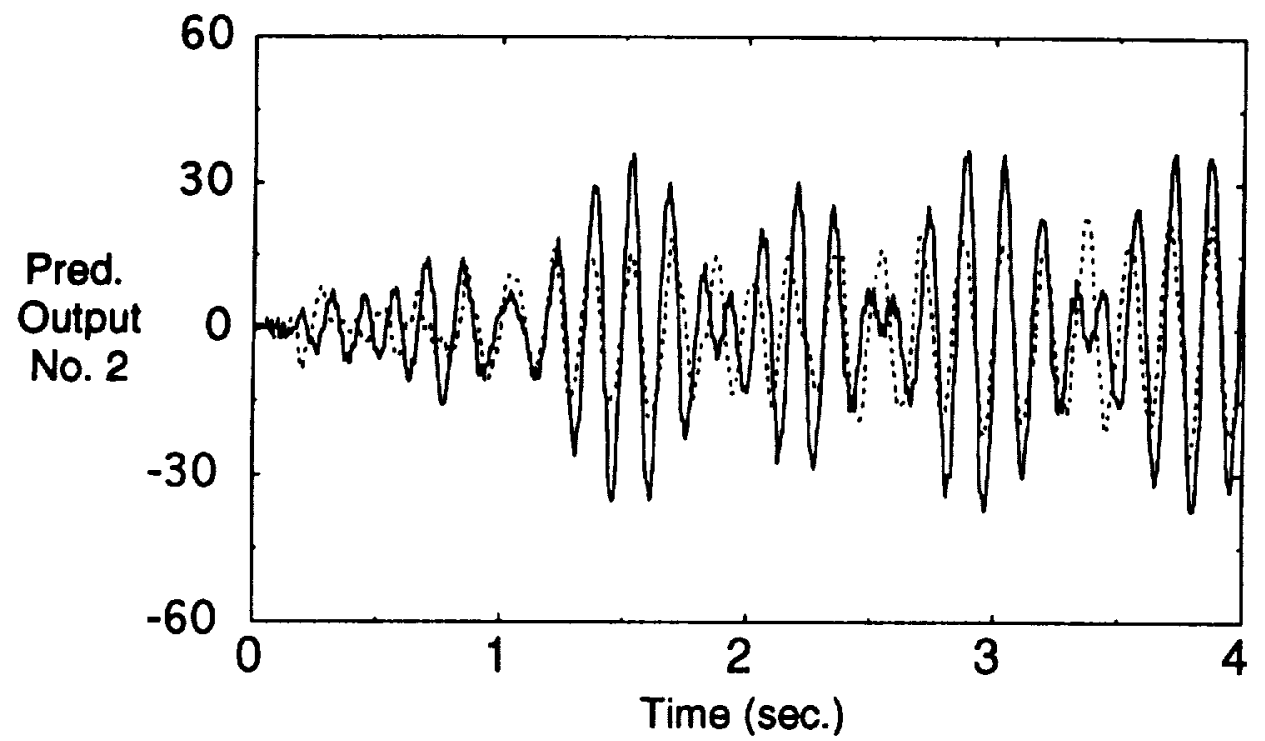

Fig. 4b: Actual vs. reconstructed response for the second output (- Test, - - Reconstruction): Backward OKID via an ARX model of order $p=10$ with 1000 data points. 


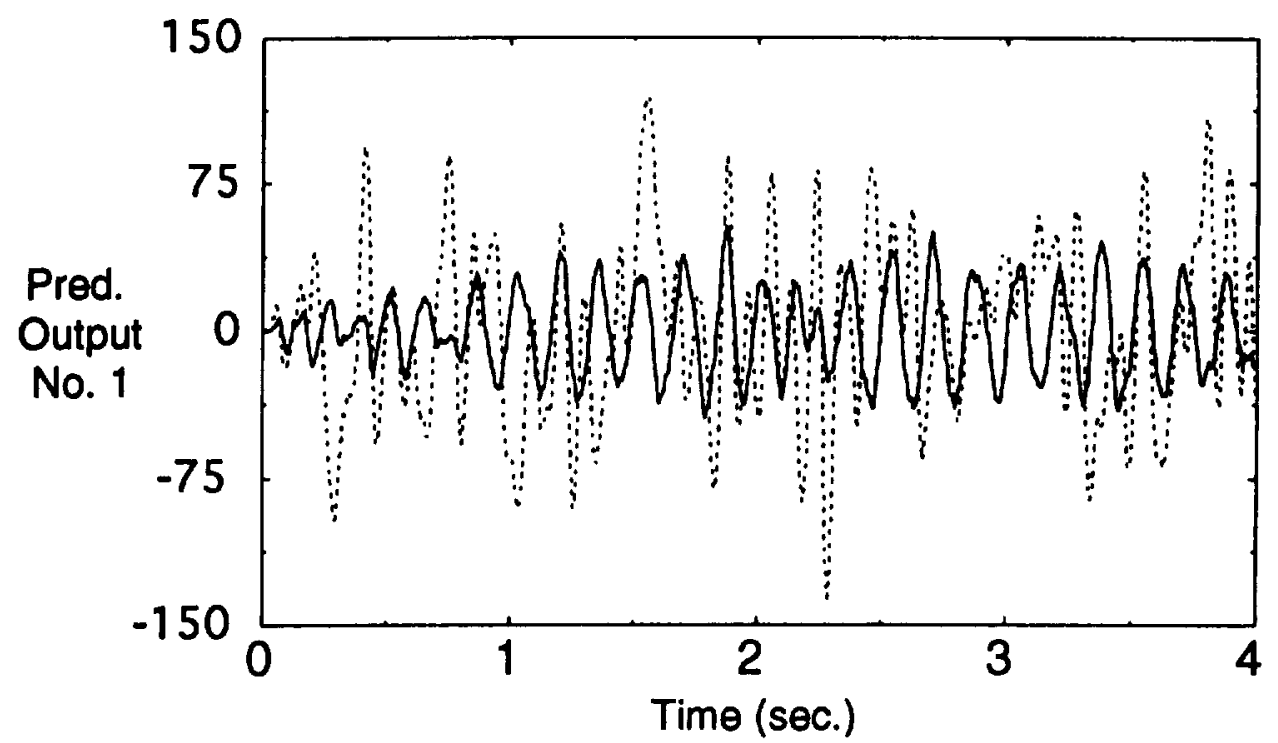

Fig. 5a: Actual vs. reconstructed response for the first output (- Test, - - Reconstruction): Backward OKID via an ARMAX model of order $p=10$ with 1000 data points after one cycle of iteration.

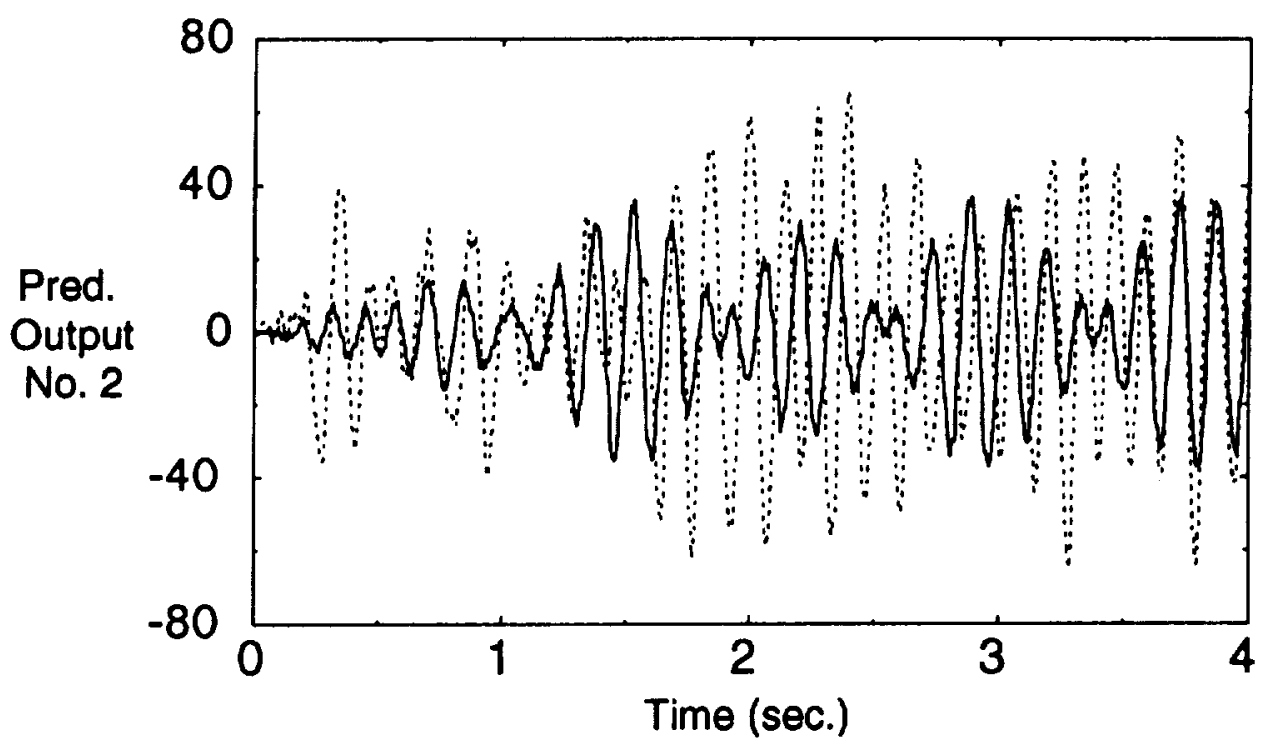

Fig. 5b: Actual vs. reconstructed response for the second output (- Test, - - Reconstruction): Backward OKID via an ARMAX model of order $p=10$ with 1000 data points after one cycle of iteration. 


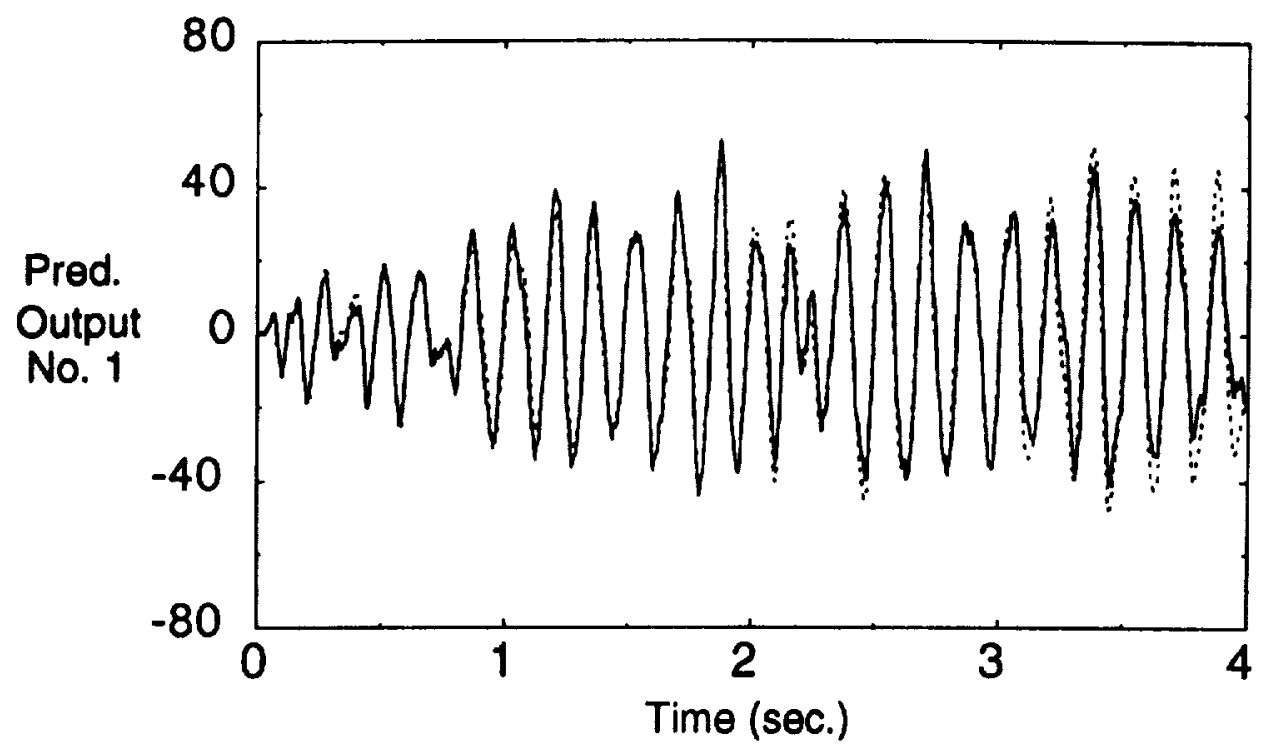

Fig. 6a: Actual vs. reconstructed response for the first output (- Test, - - Reconstruction): Backward OKID via an ARMAX model of order $p=10$ with 1000 data points after two cycles of iterations.

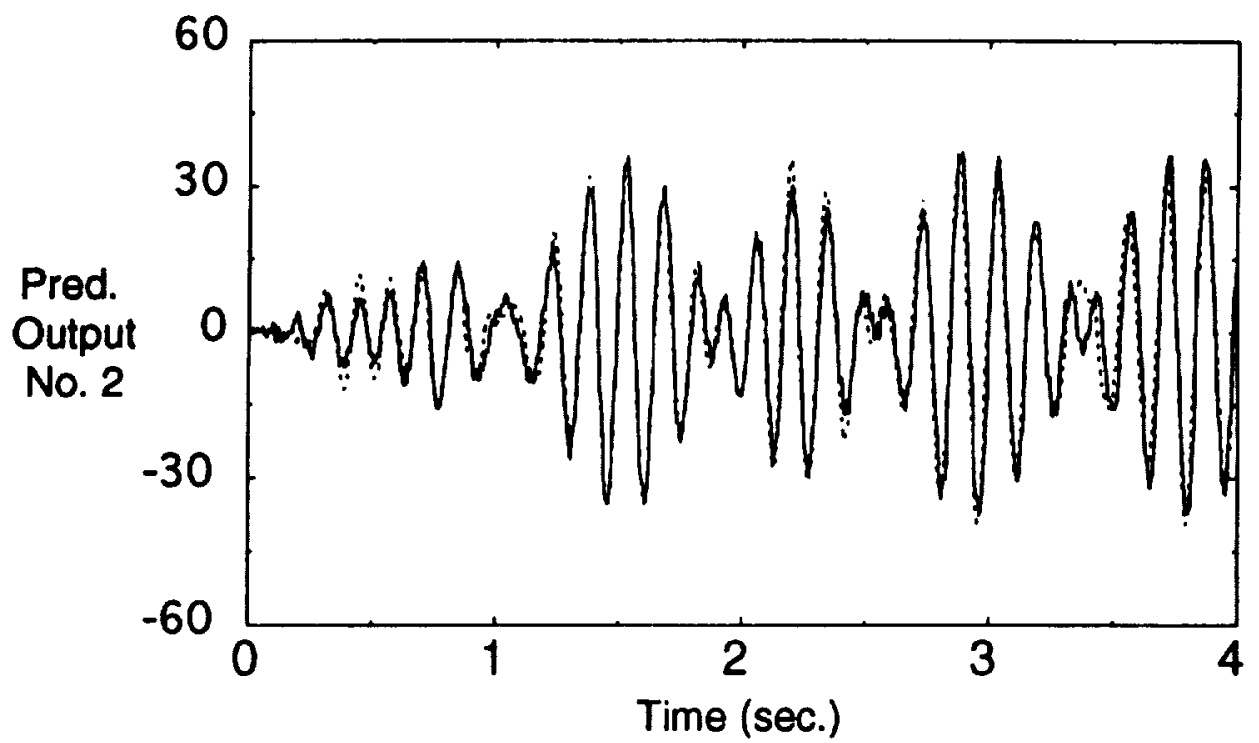

Fig. 6b: Actual vs. reconstructed response for the second output (- Test, - - Reconstruction): Backward OKID via an ARMAX model of order $p=10$ with 1000 data points after two cycles of iterations. 

Public reporting burden for this collection of information is estimated to average 1 hour per response, including the time for reviewing instructions, searching existing data sources. gathering and maintaining he data needed, and completing and reviewing collection of riformation. Send comments regarding this burden estimate or any other aspect of this Davis Highway Sute 204 . Arlington, VA 222024302 and to the Otfice of Management and Budget, Paperwor (0)

\begin{tabular}{|l|l|l} 
1. AGENCY USE ONLY (Leave blank) & $\begin{array}{c}\text { 2. REPORT DATE } \\
\text { June } 1992\end{array}$ & $\begin{array}{l}\text { 3. REPORT TYPE AND DATES COVERED } \\
\text { Technical Memorandum }\end{array}$
\end{tabular}
4. TITLE AND SUBTITLE
Linear System Identification via Backward-Time

Observer Models

5. FUNDING NUMBERS

6. AUTHOR(S)

Jer-Nan Juang and Minh Q. Phan

7. PERFORMING ORGANIZATION NAME(S) AND ADORESS(ES)

NASA Langley Research Center

Hampton, VA 23665-5225

8. PERFORMING ORGANIZATION REPORT NUMBER

\section{SPONSORING/MONITORING AGENCY NAME(S) AND ADDRESS(ES)}

10. SPONSORING / MONITORING AGENCY REPORT NUMBER

National Aeronautics and Space Administration Washington, DC 20546-0001

NASA TM-107632

\section{SUPPLEMENTARY NOTES}

Juang: NASA Langley Research Center, Hampton, VA; Phan: Lockheed Engineering \& Sciences Co., Hampton, VA

Unclassified--Unlimited

Subject Category 39

\section{ABSTRACT (Maximum 200 words)}

This paper presents an algorithm to compute the Markov parameters of a backward-time observer for a backward-time model from experimental input and output data. The backward-time observer Markov parameters are decomposed to obtain the backward-time system Markov parameters (backward-time pulse response samples) for the backward-time system identification. The identified backward-time system Markov parameters are used in the Eigensystem Realization Algorithm to identify a backward-time state-space model, which can be easily converted to the usual forward-time representation. If one reverses time in the model to be identified, what were damped true system modes become modes with negative damping, growing as the reversed time increases. On the other hand, the noise modes in the identification still maintain the property that they are stable. The shift from positive damping to negative damping of the true system modes allows one to distinguish these modes from noise modes. Experimental results are given to illustrate when and to what extent this concept works.

\section{SUBECT TERMS}

System Identification, Modal Parameter Identification, Observer Identification

\begin{tabular}{l|l}
\hline 17. SECURITY CLASSIFICATION & 18. SECURITY CLASSIFICATION \\
OF REPORT & OF THIS PAGE \\
Unlcassified & Unclassified
\end{tabular}

19. SECURITY CLASSIFICATION OF ABSTRACT
15. NUMBER OF PAGES 25

16. PRICE CODE $\mathrm{A03}$

20. LIMITATION OF ABSTRACT

Standard Form 298 (Rev. 2-89) Prescribed by ANSI Std. 239.18 298.102 

